\title{
Prediction of welding shrinkage deformation of bridge steel box girder based on Wavelet Neural Network
}

\author{
Yulong Tao ${ }^{1, \text { a) }}$, Yunshui Miao ${ }^{2, \text { b) }}$ Jiaqi Han ${ }^{1, c)}$ and Feiyun Yan ${ }^{3, \text { d) }}$ \\ ${ }^{1}$ School of Dalian University of Technology, Panjin 124000, China. \\ ${ }^{2}$ School of Tongda college of Nanjing university of posts and telecommunications ,Yangzhou210003 China. \\ ${ }^{3}$ School of North University of China, Shuozhou 036000, China. \\ a)18342781127@163.com \\ b)515617762@qq.com \\ c)1986064051@qq.com \\ d)jqh19970107@163.com
}

\begin{abstract}
Aiming at the low accuracy of traditional forecasting methods such as linear regression method, this paper presents a prediction method for predicting the relationship between bridge steel box girder and its displacement with wavelet neural network. Compared with traditional forecasting methods, this scheme has better local characteristics and learning ability, which greatly improves the prediction ability of deformation. Through analysis of the instance and found that after compared with the traditional prediction method based on wavelet neural network, the rigid beam deformation prediction accuracy is higher, and is superior to the BP neural network prediction results, conform to the actual demand of engineering design.
\end{abstract}

Keywords: Bridge Steel Box Girder, Wavelet Neural Network, Prediction Method

\section{INTRODUCTION}

In recent years, the flat steel box girder with superior wind resistance has been widely used as the stiffening beam of the long-span cable supported suspension bridge and the cable-stayed bridge. [1]. It is an important problem in engineering to realize the dynamic detection of the bridge steel box girder and to predict the deformation and improve the related performance.

When the required accuracy of bridge rigid box is not high, we think the trend of shrinkage deformation of steel box girder is regular with time. We can use linear regression to do the problem study.when ship accuracy requirement is not high, we can using the mathematical statistics method and other classical regression prediction, Spraragen and Ettinger put on carbon steel butt joints transverse shrinkage formula $\mathrm{Sb}=0.2 \mathrm{~A} / \mathrm{t}+0.05 \mathrm{~d}$ [2] (A weld cross-sectional area, mm2; t mm; D of plate thickness, welding gap volume, mm); Liu Yujun, Li Yanjun [3] in the establishment of the forecast model of the BP neural network is introduced into the model, the complex mechanism, input and nonlinear response significantly under the condition of obvious advantages, solve the shrinkage problem.

On the basis of the reanalysis of the factors affected by the rigid box of the bridge, we use the wavelet neural network is used to predict the welding shrinkage deformation of the bridge rigid box.

\section{INTRODUCTION OF WAVELET NEURAL NETWORK}

According to the different combination of wavelet analysis and neural network form can be divided into "loose" and "tight" type two, "loose" refers to the use of wavelet analysis of signal preprocessing, and then input the neural network processing, "embedded" refers to the excitation function of neurons in the hidden layer containing nerve substitute network with wavelet function,

Fully inherit the good time frequency localization of the wavelet transform and the characteristics of the self-learning function of the neural network, it is widely used.

It is used in the fields of signal processing, data compression, pattern recognition and fault diagnosis. [4] this paper is based on the wavelet neural network to predict the shrinkage deformation of the rigid beam of the 
bridge.

The wavelet neural network is built by replacing the excitation function of hidden layer with wavelet function, retaining the characteristics of BP neural network's error propagation.Its basic structure is as in the Figure 1.

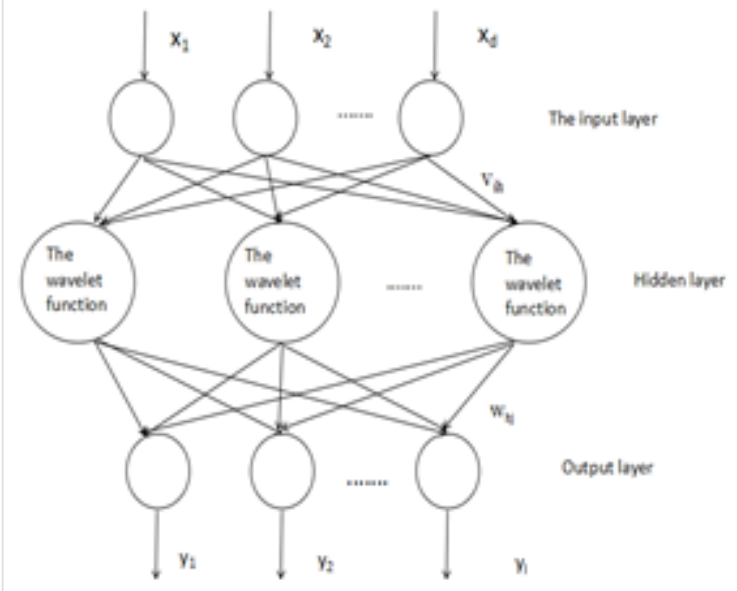

FIGURE 1. Neural network structure

TABLE 1. The meaning of symbols

\begin{tabular}{|c|c|}
\hline Symbol & Stand for \\
\hline$\theta_{\mathrm{j}}$ & The threshold value of the $j$ neuron in the output layer. \\
\hline$\lambda_{\mathrm{n}}$ & The threshold value of the $h$ neuron in the hidden layer. \\
\hline $\mathrm{v}_{\text {in }}$ & $\begin{array}{l}\text { The connection of the } i \text { neuron of the input layer and the } \\
\qquad h \text { neuron of the hidden layer. }\end{array}$ \\
\hline $\mathrm{w}_{\mathrm{hj}}$ & $\begin{array}{l}\text { The connection right of the } J \text { neuron in the } h \text { neuron and } \\
\text { the output layer of the hidden layer }\end{array}$ \\
\hline $\mathrm{h}_{\mathrm{j}}$ & The wavelet function in the hidden layer. \\
\hline $\mathrm{a}_{\mathrm{j}}$ & Scaling factor \\
\hline $\mathrm{b}_{\mathrm{j}}$ & Translation factor \\
\hline $\mathrm{N}$ & The number of hidden layers. \\
\hline
\end{tabular}

\section{STRUCTURE OF WAVELET NEURAL NETWORK.}

\section{The Selection of wavelet functions}

The function of the hidden layer node is to extract and store its inherent law from the sample. Each of the hidden layer nodes has several weights, and each weight value is the parameter to enhance the network mapping capability. The number of hidden layer nodes is too small, and the ability of network to obtain information from samples is poor, which is not enough to generalize and reflect the sample rule of training concentration. The number of hidden layer nodes is too large, and it is possible to keep in mind the regular contents of the samples, so that the so-called "over-fitting" problem can reduce the generalization ability of the network. 
we choose Morlet wavelet as the the research object:

$$
\phi=\cos (1.75 x) e^{\frac{-x^{2}}{2}}
$$

Compared to other functions, the Morlet wavelet basis function is symmetric and the expression is clear and can be applied well.

the output of the $\mathrm{j}$ neuron node is:

$$
\mathrm{f}(\mathrm{j})=\phi\left(\frac{\sum_{i=1}^{d} w_{i} x_{i}-b_{i}}{\mathrm{a}_{\mathrm{j}}}\right) \quad(j=1,2 \ldots m)
$$

The output of the $\mathrm{k}$ neuron node in the output layer is:

$$
\mathrm{y}(\mathrm{k})=\sum_{j=1}^{N} \gamma_{j \mathrm{k}} \mathrm{f}(j)
$$

\section{The determination of the number of hidden layers}

In theory, increase the number of hidden layer can reduce the error of the wavelet neural network, improve the accuracy of prediction, but when the number of hidden layer is too large, will increase the complexity of the structure and operation, greatly increase the learning time. For any continuous function within the closed interval, the BP network of an implicit layer can be used to approximate.

\section{THE NUMBER OF HIDDEN LAYER NODES.}

The node selection of hidden layer is a more complex problem, and the empirical formulas in the literature are more errors, which often need to be determined by a large number of experiments on the basis of experience.

The function of the hidden layer node is to extract and store its inherent law from the sample. Each of the hidden layer nodes has several weights, and each weight value is the parameter to enhance the network mapping capability. The number of hidden layer nodes is too small, and the ability of network to obtain information from samples is poor, which is not enough to generalize and reflect the sample rule of training concentration. The number of hidden layer nodes is too large, and it is possible to keep in mind the regular contents of the samples, so that the so-called "over-fitting" problem can reduce the generalization ability of the network.

\section{WAVELET NEURAL NETWORK ALGORITHM}

The specific steps of wavelet neural network algorithm are as follows:

A、 Initialization of wavelet neural network.

The purpose of this process is to give the translation factor, the weight of each connection first value.

B、Input of training samples.

The process inputs network learning samples $X=\left(x_{1}, x_{2} \ldots \ldots x_{m}\right)$ and corresponding expected outputs $\mathrm{d}_{\mathrm{n}}^{\mathrm{p}}$.

C、 Self-learning of the network.

D、 Calculate the prediction error.

The error in the prediction process is calculated by the formula.

$$
\mathrm{e}=\sum_{k=1}^{n} \mathrm{~d}_{n}^{p}-y_{n}^{p}
$$

E、 correction parameters

The error function is reduced by the gradient descent method, and the corresponding parameters of the 
network connection weight and wavelet function are corrected.

The $\mathrm{n}$ neuron of the output layer is expected to be $\mathrm{d}_{n}^{\mathrm{d}}$, and the actual output is $\mathrm{y}_{n}^{\mathrm{d}}$, and the error function is:

$$
E=\frac{1}{2 \mathrm{~d}} \sum_{d=1}^{d} \sum_{i=1}^{l}\left(d_{n}^{d}-y_{n}^{\mathrm{p}}\right)
$$

The learning process of wavelet neural network can be understood as the process of minimizing the error function value $\mathrm{E}$.

For network connection weight $\omega_{\mathrm{ij}}$, its descent gradient.

$$
\partial_{\mathrm{ij}}=\frac{\partial E_{\mathrm{d}}^{p}}{\partial \omega_{\mathrm{ij}}}=\left(d_{n}^{p}-y_{n}^{p}\right) y_{n}^{p}\left(1-y_{n}^{p}\right)
$$

As a result, the weight $\omega_{\mathrm{ij}}$ can be adjusted to:

$$
\omega_{\mathrm{ij}}(t+1)=\omega_{i j}(t)+\eta \sum_{d=1}^{D} \partial_{i j}+\lambda \Delta_{1} w_{i j}(t)
$$

The remaining parameters can be modified according to the same principle.

F、 determine whether the algorithm is over.

According to the error of the previous step, whether the accuracy is in accordance with the requirements, if it is consistent, stop learning, or go back to the second step to study again.

\section{EXAMPLE ANALYSIS}

We choose a bridge with a main span of 1088 meters and the main beam for 140 segments as the analysis object. According to the data that has been completed at the scene, 15 weld data of roof are selected as the research object respectively.The predicted results are shown in Table 3 and Figure 2.

TABLE 2. Sample data sheet

\begin{tabular}{|c|c|c|c|c|}
\hline Sample no. & $\begin{array}{c}\text { Atmospheric } \\
\text { temperature }\end{array}$ & $\begin{array}{c}\text { Roof } \\
\text { temperature }\end{array}$ & $\begin{array}{c}\text { Ceiling } \\
\text { welding feet } \\
\text { wide }\end{array}$ & $\begin{array}{c}\text { Roof deformation } \\
\text { width }\end{array}$ \\
\hline 1 & 7.2 & 3.2 & 7.4 & -2.9 \\
\hline 2 & 6 & 2.7 & 7.8 & -2.7 \\
\hline 3 & 6 & 2.7 & 8.2 & -2.72 \\
\hline 4 & 1.7 & -3.8 & 8.2 & -1.64 \\
\hline 5 & 7.2 & 3.2 & 8.6 & -2.85 \\
\hline 6 & 8 & 1.2 & 5.8 & -2.14 \\
\hline 7 & 4.6 & 1.9 & 9 & -2.12 \\
\hline 8 & 8 & 1.3 & 7 & -2.51 \\
\hline 9 & 6 & 2.7 & 8 & -2.7 \\
\hline 10 & 4.6 & 1.9 & 7.4 & -2.02 \\
\hline 11 & 4.6 & 1.9 & 9.2 & -2.1 \\
\hline 12 & 2.1 & -4.4 & 8.4 & -2.12 \\
\hline 13 & 3.4 & -1 & 6.9 & -1.86 \\
\hline 14 & 1.7 & -3.8 & 8.1 & -1.46 \\
\hline 15 & 4.6 & 1.9 & 8.6 & -2.09 \\
\hline
\end{tabular}


TABLE 3. Error comparison of two prediction methods.

\begin{tabular}{|c|c|c|c|c|c|}
\hline Sample no & Deformatio & \multicolumn{2}{|c|}{ BP neural network } & \multicolumn{2}{c|}{ Wavelet neural network. } \\
\cline { 3 - 6 } & $\begin{array}{c}\text { n measured } \\
\text { value. }\end{array}$ & $\begin{array}{c}\text { Predictive } \\
\text { value }\end{array}$ & error & $\begin{array}{c}\text { Predictive } \\
\text { value }\end{array}$ & error \\
\hline 13 & -1.86 & -1.8 & $3.2 \%$ & -1.83 & $1.63 \%$ \\
\hline 14 & -1.46 & -1.51 & $3.42 \%$ & -1.48 & $1.37 \%$ \\
\hline 15 & -2.09 & -2.13 & $1.91 \%$ & -2.11 & $0.95 \%$ \\
\hline
\end{tabular}

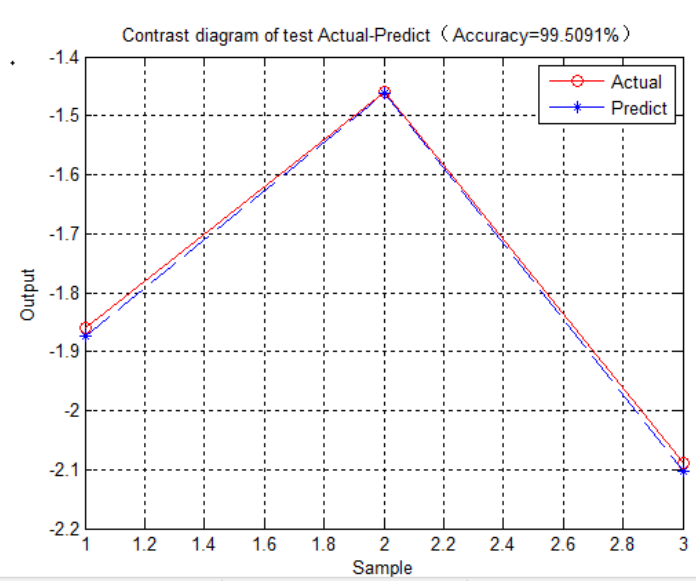

FIGURE 2. Deviation of BP neural network.

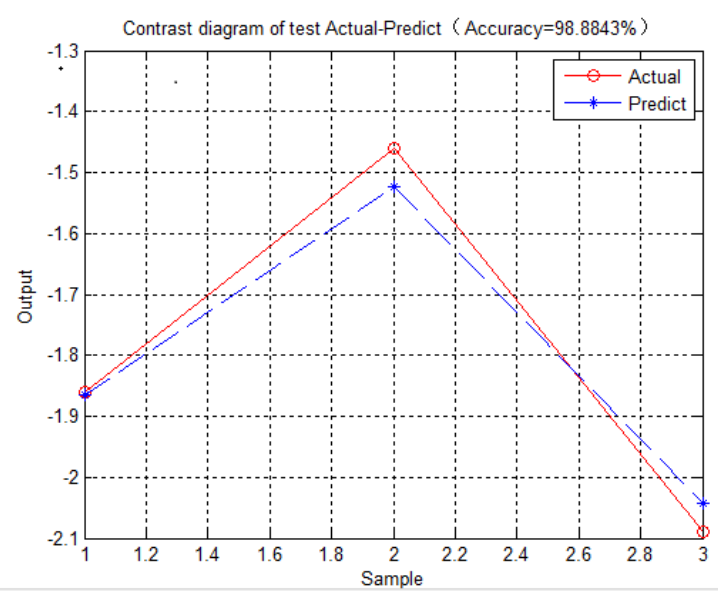

FIGURE 3.The deviation of the wavelet neural network.

\section{SUMMARY}

The deformation prediction of bridge steel box girder with wavelet neural network is more suitable for engineering practice than traditional method. The introduction of this method can not only improve the accuracy of prediction, but also predict the local learning ability of wavelet function.

\section{REFERENCES}

1. Bai ling, shi zhiqiang, shi yongji. Welding shrinkage deformation and control of large steel box girders [J]. Steel structure. 9-11 (53)

2. Zeng yuanxing. Welding structure analysis [M]. Zhang weichang. Beijing: mechanical industry press, 1985

3. Liu yujun, li yanjun. Modeling of shrinkage of Marine steel welding by BP neural network [J]. Journal of dalian university of technology.2004; (4) : 533-533

4. Zuo dongguang, zhou shuai, zhang xinyu. Xiaobo neural network [J]. Journal of sichuan military engineering. 2012. : 85-87. 